\title{
Jeder Zweite weiß nicht, wo sein Herz ist
}

\author{
Dr. med. \\ Dirk Einecke \\ Chefredakteur
}

\section{PATIENTEN SIND OFT ERSTAUNLICH UNWISSEND}

\section{Der Körper als Terra incognita}

Eigentlich sollte man ja meinen, dass sich angesichts der medialen Dauerberieselung mit Gesundheitsthemen und unzähligen Arzt- und Krankenhausserien das anatomische Wissen von Otto Normalverbraucher inzwischen dem Niveau eines Medizinstudenten im vierten Semester angenähert hat. Weit gefehlt: In einer englischen Studie wurden gut 700 Teilnehmern Bilder des menschlichen Körpers vorgelegt, auf denen bestimmte Bereiche schattiert dargestellt waren. Diesen Bereichen sollten sie aus einer
Liste die entsprechenden Organe zuordnen.

Das Ergebnis: 86\% konnten zwar den Darm richtig zuordnen und $81 \%$ die Blase, die korrekte Position der Lunge fanden aber nur $31 \%$ und das Herz hatten nur $46 \%$ am rechten Fleck. Insgesamt war nur etwa die Hälfte der Zuordnungen richtig.

Fazit für die Praxis: Scheuen Sie sich nicht, im Patientengespräch auch scheinbar Banales zu erklären.

Weinmann J. et al. BMC Family Practice, Juni 2009

\section{BLUTHOCHDRUCK, ÜBERGEWICHT}

\section{Die Gefahren der kurzen Nachtruhe}

Wer regelmäßig wenig Schlaf abbekommt, hat ein deutlich erhöhtes Hypertonierisiko. Das belegt eine Studie, in der Schlafgewohnheiten und Blutdruckwerte von 578 Personen über fünf Jahre hinweg registriert wurden. Die Studienautoren empfehlen daher, mindestens sieben Stunden zu schlafen. Ob eine solche Verlängerung der Schlafdauer bei Hypertonikern den Blutdruck senken kann, wird derzeit in einer größeren Studie untersucht. Schlafmangel scheint auch die Entstehung von Übergewicht zu begünstigen, wie eine Laborstudie mit 92 Probanden ergab. Es wird diskutiert, ob der Schlafentzug die Gier nach Kohlenhydraten weckt oder einfach mehr Zeit zum Essen lässt.

Arch Intern Med 2009;169:1055-61; Kongress der American Academy of Sleep Medicine, Juni 2009

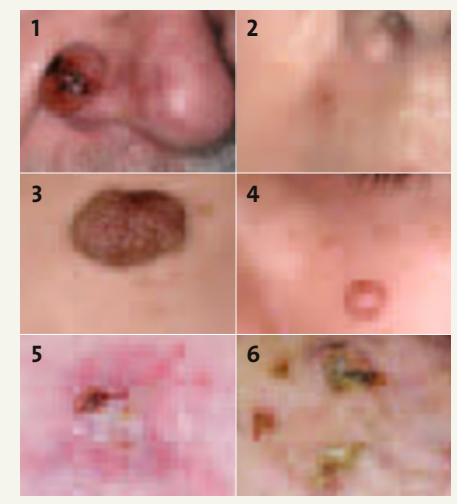

Titelbild: K. Gauwerky $(1,2,5,6)$; H. Schulz $(3,4)$

\section{Bösartig?}

1, 2 Basalzellkarzinome

3 Papillomatöser Naevus

4 Fibrosierter Naevuszellnaevus

5 Basaliom

6 Plattenepithelkarzinom

Ihren diagnostischen Blick schärfen und auf die Probe stellen können Sie mit unserer CME-Fortbildung ab Seite 39. Übrigens eine der letzten Gelegenheiten, Ihr CME-Konto auf den geforderten Stand von 250 Punkten zu bringen.

\section{FAST FOOD ALS AUSLÖSER?}

\section{Darmkrebs bei jungen Menschen nimmt zu}

Insgesamt ist in den USA die Zahl der Darmkrebserkrankungen in den letzten zehn Jahren zurückgegangen. Experten sehen darin einen Erfolg der Screeningprogramme und damit der Möglichkeit, Malignomvorstufen frühzeitig zu entfernen. Auf der anderen Seite hat die Darmkrebsrate bei den 20- bis 49-Jährigen in derselben Zeit kontinuierlich zugenommen. Es wird vermutet, dass der seit den 1970er-Jahren stark angestiegene FastFood-Konsum (z. B. mehr rotes und verarbeitetes Fleisch, weniger Milchprodukte und Ballaststoffe) zu diesem Anstieg beigetragen hat.

Cancer Epidemiol Biomarker Prev 2009;18:1695-98

\section{SEXUELLE DYSFUNKTION}

\section{Macht Licht Lust?}

Die Zirbeldrüse kann einen hemmenden Effekt auf den Sexualtrieb ausüben. Italienische Forscher haben jetzt geprüft, ob eine Inhibition der Zirbeldrüsenaktivität sich positiv auf den Sexualtrieb auswirkt. Dazu wählten sie neun Männer mit primärer sexueller Dysfunktion aus, bei denen keine organischen oder psychiatrischen Erkrankungen und kein Medikamenten- oder Drogenabusus bestanden.

Die Probanden sollten sich nach dem Erwachen 30 Minuten vor eine Lichtquelle setzen, die weißes Licht mit 10000 Lux (Verum) bzw. nur 100 Lux (Placebo) abgab. Nach zwei Wochen Therapie hatten drei der fünf Probanden, die die volle Lichtquelle erhalten hatten, ihre sexuelle Dysfunktion überwunden. In der Placebogruppe wurde keine Veränderung des Sexualverhaltens beobachtet.

Psychotherapy and Psychosomatics 2009;78:127 